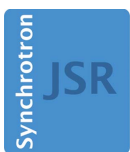

JOURNAL OF SYNCHROTRON RADIATION

ISSN 1600-5775

Received 28 September 2018

Accepted 11 February 2019

Edited by I. Schlichting, Max Planck Institute for Medical Research, Germany

${ }^{1}$ This article will form part of a virtual special issue on X-ray free-electron lasers.

\# Current address: Paul Scherrer Institut, 5232 Villigen PSI, Switzerland.

Keywords: time-resolved molecular dynamics; non-linear light-matter interaction; reaction microscope; multi-particle coincidence spectroscopy; free-electron laser; XUV-XUV pump-probe spectroscopy.

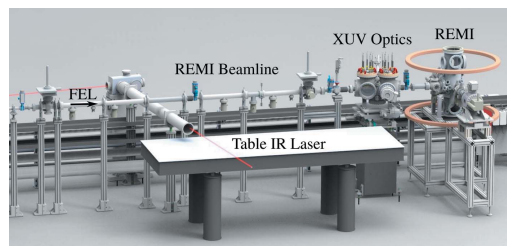

(C) 2019 International Union of Crystallography

\section{Reaction microscope endstation at FLASH2 ${ }^{1}$}

\author{
Georg Schmid, ${ }^{a} *$ Kirsten Schnorr, ${ }^{a} \neq$ Sven Augustin, ${ }^{a}$ Severin Meister, ${ }^{a}$ \\ Hannes Lindenblatt, ${ }^{a}$ Florian Trost, ${ }^{a}$ Yifan Liu, ${ }^{a}$ Markus Braune, ${ }^{b}$ Rolf Treusch, \\ Claus Dieter Schröter, ${ }^{a}$ Thomas Pfeifer $^{\mathrm{a}}$ and Robert Moshammer ${ }^{\mathrm{a} *}$ \\ aMax-Planck-Institut für Kernphysik, Saupfercheckweg 1, 69117 Heidelberg, Germany, and \\ ${ }^{\mathbf{b}}$ Deutsches Elektronen-Synchrotron DESY, Notkestrasse 85, 22607 Hamburg, Germany. \\ *Correspondence e-mail: georg.schmid@mpi-hd.mpg.de, robert.moshammer@mpi-hd.mpg.de
}

A reaction microscope dedicated to multi-particle coincidence spectroscopy on gas-phase samples is installed at beamline FL26 of the free-electron laser FLASH2 in Hamburg. The main goals of the instrument are to follow the dynamics of atoms, molecules and small clusters on their natural time-scale and to study non-linear light-matter interaction with such systems. To this end, the reaction microscope is combined with an in-line extreme-ultraviolet (XUV) split-delay and focusing optics, which allows time-resolved XUV-XUV pumpprobe spectroscopy to be performed.

\section{Introduction}

A complete picture of the formation, internal rearrangement and the break-up of a molecule is only obtained if the evolution of the reaction is observed in a time-resolved manner (Zewail, 2000). As underlying processes like charge migration and energy redistribution within the molecule typically happen within a few to tens of femtoseconds, a spectroscopic technique that is fast enough to record the reaction is needed (Zewail, 1988). With the invention of shortwavelength free-electron lasers (FELs) (Ackermann et al., 2007), the research area of atomic, molecular and optical (AMO) physics obtained a new experimental tool that provides femtosecond light pulses of extreme-ultraviolet (XUV) photons at unprecedented intensities (Feldhaus et al., 2013; Yabashi et al., 2013; Callegari et al., 2016). The unique properties of XUV FEL pulses allow XUV pump-probe spectroscopy to be employed to study dynamical processes like isomerization (Jiang et al., 2010a), nuclear wave-packet oscillations in molecules (Jiang et al., 2010b; Magrakvelidze et al., 2012), interatomic Coulombic decay (Schnorr et al., 2013), electron rearrangement in dissociating molecules (Schnorr et al., 2014), the expansion of clusters (Krikunova et al., 2012; Sauppe et al., 2018), non-equilibrium transient states in functional solids (Pontius et al., 2018) and attosecond interferometry (Usenko et al., 2017). Furthermore, shortwavelength FELs open up a new regime in non-linear lightmatter interaction, e.g. multi-photon ionization can now be studied at XUV photon energies (Sorokin et al., 2007b; Moshammer et al., 2007; Richter et al., 2009; Hikosaka et al., 2010; Gerken et al., 2014).

Besides an appropriate light source, a detection apparatus, which extracts the essential information on the process under investigation, is decisive.

Reaction microscopes (REMIs) are state-of-the-art coincidence momentum imaging spectrometers to resolve and 


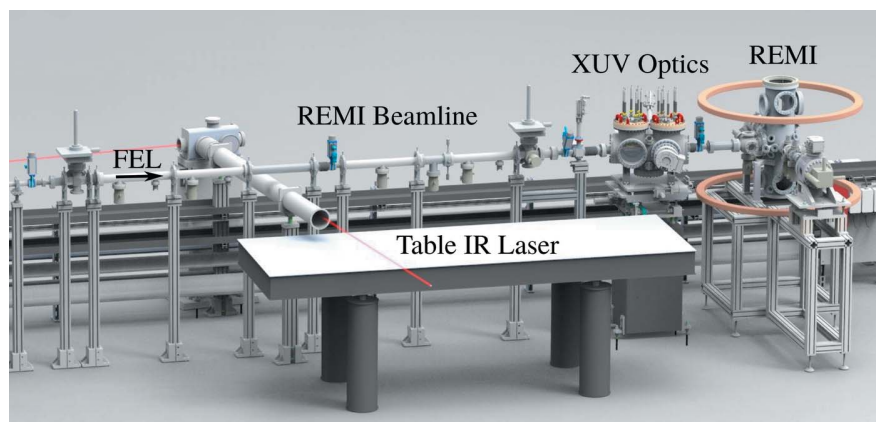

Figure 1

Overview of the REMI endstation at FL26. The FEL beam enters from the left. Shown are the REMI ( $c f$. Section 2), the in-line XUV split-delay and focusing optics ( $c f$. Section 3), the REMI beamline section ( $c f$. Section 4) and an optical table for the IR laser (cf. Section 6). The figure is taken from Schmid (2018).

study fundamental reactions in atoms or molecules, triggered upon collisions with ions, the impact of electrons or absorption of photons (Ullrich et al., 2003).

Taking advantage of the synergy arising from the combination of a REMI and a high-repetition-rate XUV FEL (Rudenko et al., 2010; Moshammer \& Schnorr, 2014), a dedicated user endstation is installed at beamline FL26 of the free-electron laser FLASH2 (Plönjes et al., 2016; Faatz et al., 2016). Fig. 1 shows the layout of the endstation. In this paper, the main components are introduced and commissioning results are discussed.

\section{Reaction microscope}

Reaction microscopes are multi-particle coincidence spectrometers for AMO physics, which allow measuring the initial energies and emission angles of the charged products, i.e. ions and electrons, of fundamental atomic and molecular reactions in the gas phase (Moshammer et al., 1996; Dörner et al., 2000).

To this end, the charged reaction fragments, i.e. atomic or molecular ions and electrons, are accelerated onto large-area time- and position-sensitive detectors. By measuring the timeof-flight (TOF) and the impact position of the particles on the detectors, the initial three-dimensional momentum vectors can be reconstructed. While the ions carry information about the nuclear dynamics during a reaction, the ejected electrons are sensitive to the electronic structure of the system under investigation. In the ideal case, electrons and ions are measured in coincidence and, by applying momentum conservation, fragments emerging from a single atom or molecule can be selected. This enables so-called kinematically complete experiments to be performed (Kurka et al., 2009).

The REMI installed at FLASH2 is schematically shown in Fig. 2. The FEL beam (purple) is focused into a supersonic gas jet ( $c f$. Section 2.1), which contains the target atoms, molecules or clusters (green spheres). Created ions (red trajectory) and electrons (blue trajectory) are separated by their different charges and guided onto opposing detectors ( $c f$. Section 2.3) by the electric field of the spectrometer ( $c f$. Section 2.2). The magnetic field generated by a pair of Helmholtz coils forces

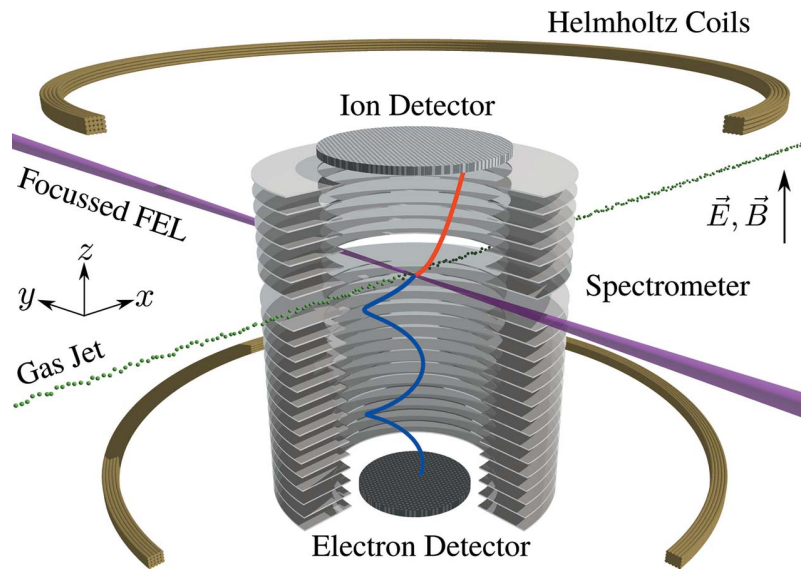

Figure 2

Schematic drawing of the REMI. Shown are the main components, i.e. spectrometer, ion detector, electron detector and a pair of Helmholtz coils. The FEL beam (purple) is focused into a supersonic gas jet (green spheres). Created ions (red trajectory) and electrons (blue trajectory) are guided by a combination of an electric (E) and a magnetic field (B) onto their respective detector. The coordinate system $(x, y, z)$ used within this section is also shown. The figure is adapted from Schmid (2018).

the electrons in cyclotron orbits on their way to their detector. The combination of a homogeneous electric and magnetic field ensures a solid-angle acceptance of $4 \pi$ for all ions and electrons of interest. In the following, the key components of the REMI are introduced.

\subsection{Target injection}

The target preparation is based on supersonic expansion of gas-phase samples. For this, gas is expanded through a nozzle from a high-pressure reservoir into vacuum and a supersonic jet is formed. In doing so, the undirected thermal energy of the gas particles is transformed into directed kinetic energy, which results in a decrease of internal temperature. The achieved temperature depends on several parameters, in particular on the specific gas, the nozzle diameter, the backing pressure and the temperature of the gas before the expansion (Scoles, 1988).

Three different nozzle assemblies are available at the REMI endstation. Firstly, a cooled nozzle allows to pre-cool the gas before the expansion. Cooling down to cryogenic temperatures is achieved by using a closed-cycle helium refrigerator cold-head, and very low jet temperatures are reached (for example $\sim 0.5 \mathrm{~K}$ for a helium jet). This results in very low momentum uncertainty of the target atoms or molecules. Hence, this nozzle assembly is suited for experiments that ask for a high-momentum resolution for the recoil-ions (Kurka et al. 2010). Secondly, a heatable nozzle can be installed to inject liquid and solid samples with low vapor pressure (Schnorr et al., 2014). It is based on sublimating the sample in a heatable reservoir outside of the vacuum. The molecules are then carried to the nozzle by a seeding gas or the vapor pressure. An increasing temperature gradient from the reservoir to the nozzle circumvents re-sublimation and, thus, clogging of the system. The heatable nozzle can operate up to temperatures of $200^{\circ} \mathrm{C}$. Thirdly, a nozzle assembly where the reservoir for 
liquid samples is integrated into the vacuum system is available. The design is based on evaporating the liquid as close as possible to the nozzle to prevent clogging by condensation. The system can be operated with and without a seeding gas. Using this nozzle assembly, the generation of water and water-rare-gas dimers has been accomplished (Meister, 2016).

The experimental implementation of the jet system is shown in Fig. 3. It features six vacuum stages that are differentially pumped. Hence, despite backing pressures of up to 70 bar in the nozzle, ultra-high-vacuum (UHV) conditions are preserved in the REMI interaction chamber ( $c f$. Section 2.4). In order to form a supersonic jet, the gas expands from a high-pressure reservoir (0) through the nozzle (typical diameters $5-50 \mu \mathrm{m}$ ) into the vacuum of the first jet stage (1). A sharp-edged conical skimmer made of pure copper (inner diameter: $180 \mu \mathrm{m}$ ) is coaxially opposing the nozzle to peel off gas particles from the so-called zone of silence ( $c f$. inset Fig. 3), i.e. a region where gas particles have supersonic speeds and low internal temperatures. A subsequent second skimmer (2) (inner diameter: $400 \mu \mathrm{m}$ ) geometrically selects only gas particles with a small momentum spread in the direction perpendicular to the jet-propagation direction ( $c f$. inset Fig. 3). The nozzle assemblies introduced above are mounted on a triaxial $x y z$-manipulator, which allows adjustment of the distance between the nozzle and the first skimmer in the $x$ direction as well as the position in the perpendicular $(y, z)$ plane. The coordinate system is shown in Fig. 3. The subsequent stages $(3,4,5,6)$ are separated by apertures of $2 \mathrm{~mm}$ inner diameter. Three pairs of slits (two vertical, one horizontal) in the stages 4,5 and 6 allow tuning of the jet divergence in the $(y, z)$-plane and thus the target extension along the FEL beam direction. Finally, a spatially well confined (diameter $\leq 2 \mathrm{~mm})$, dilute $\left(\leq 10^{10}\right.$ particles $\left.\mathrm{cm}^{-3}\right)$ and cold (typically $<20 \mathrm{~K}$ ) beam of atoms, molecules or clusters enters the REMI spectrometer ( $c f$. Section 2.2). The portion of the jet which is not ionized is collected in the jet dump to avoid an increase of gas pressure in the REMI interaction chamber. The REMI interaction chamber, jet dump stage (i) and jet dump stage (ii) are separated by tubes of $4 \mathrm{~mm}$ inner diameter for differential pumping.

\subsection{Spectrometer}

The REMI spectrometer is schematically depicted in Fig. 2. It consists of ring-electrodes to create a homogeneous electric field at the interaction point and between the two detectors of the REMI. The rings are separated by a distance of $10 \mathrm{~mm}$ and are made of annealed stainless steel. They have an inner diameter of $120 \mathrm{~mm}$, an outer diameter of $200 \mathrm{~mm}$ and are
$1 \mathrm{~mm}$ thick. The individual rings are electrically connected by a cascade of $100 \mathrm{k} \Omega$ resistors and a fixed voltage can be applied across the spectrometer. The inner boundaries of the rings are sharply edged towards the center to generate well defined equipotential planes. This ensures a homogeneous electric field over the entire inner volume of the spectrometer. Fine-meshed stainless steel grids (wire diameter: $30 \mu \mathrm{m}$; mesh size: $224 \mu \mathrm{m}$; transmittance: $\sim 80 \%$ ) at both ends of the spectrometer ensure a defined termination of the electric field.

The spectrometer has a total length of $275 \mathrm{~mm}$. In order to optimize the acceptance for ions with high kinetic energies, which emerge, for example, from Coulomb explosion of molecules (Schnorr et al., 2014), the ion side of the spectrometer is kept short $(94.5 \mathrm{~mm})$. The electron side is about a factor of two longer. An ion (red trajectory in Fig. 2) of mass $m$ and charge $q$ that is created at the interaction point (center of the REMI) with zero initial momentum reaches the detector after the time-of-flight

$$
\mathrm{TOF}=d\left(\frac{2 m}{q U}\right)^{1 / 2} \propto\left(\frac{m}{q}\right)^{1 / 2} .
$$

Here, $U$ is the applied voltage and $d$ is the distance between the interaction point and the detector. The proportionality of the TOF to the square root of the mass-to-charge ratio $[c f$. equation (1)] allows different ion species to be distinguished. The spectrometer does not have a field-free drift region or, in other words, the constant electric field is active over the whole length of the spectrometer, i.e. from the ion to the electron detector. A drift region, for, for example, time focusing (Wiley \& McLaren, 1955), is not implemented since the starting point of the ions' and electrons' TOF is well defined by the small FEL focal spot diameter of $<10 \mu \mathrm{m}$ ( $c f$. Section 5.1). Furthermore, a drift region would geometrically reduce the acceptance of the ion detector for ions with high kinetic energies. 
Electrons with the same momenta as the recoiling ions move with much higher velocities compared with the heavier ions $\left(m_{\text {proton }} / m_{\mathrm{e}} \simeq 1836\right)$. With high probability they would leave the spectrometer transversally and miss the electron detector. To avoid this, a magnetic field is applied parallel to the electric field. In the magnetic field electrons fly in cyclotron orbits to their detector (blue trajectory in Fig. 2). The magnetic field (of the order of $B \simeq 10$ Gauss) is generated by a pair of Helmholtz coils ( $c f$. Fig. 2). As the cyclotron frequency is given by $\omega=q B / m$, the trajectories of the heavier ions are not measurably changed by the magnetic field.

As ions from Coulomb explosions can have high kinetic energies, high electric fields of up to $100 \mathrm{~V} \mathrm{~cm}^{-1}$ must be applied to the spectrometer to achieve full angular acceptance for these ions. The actual acceptance depends on several parameters, in particular charge state and mass of the ions, as well as the velocity offset that the particles already have as they are contained in a propagating gas jet (cf. Section 2.1) before interacting with the FEL. Taking typical values for these parameters, the spectrometer has at least a full angular acceptance for ions with kinetic energies of up to $40 \mathrm{eV}$. In order to measure electrons and ions in coincidence with sufficient resolution, lower electric fields $\left(\leq 10 \mathrm{~V} \mathrm{~cm}^{-1}\right)$ need to be applied. In this operation mode, the spectrometer typically has a full angular acceptance for electrons with maximum kinetic energies of about $40 \mathrm{eV}$.

\subsection{Detectors}

Large-area micro-channel plate (MCP) detectors are used to measure the TOF and the impact position of ions and electrons. Each detector is equipped with a stack of MCPs (Wiza, 1979), which generates the signals and allows the TOF information to be extracted. A delay-line anode (Lampton et al., 1987) located behind the MCPs is used to obtain the position information. Fig. 4 gives an overview of the detector assembly and its working principle (Jagutzki et al., 2002b).

An impinging primary particle (ion or electron) initiates an electron avalanche in a channel of the MCPs. This leads to a drop in the voltage over the MCPs, which gives a signal [ $c f$. Fig. 4(b)]. The TOF is measured relative to the FEL trigger: TOF $=t_{\mathrm{MCP}}-t_{\text {trigger }}$. The signal of $t_{\mathrm{MCP}}$ is read out capacitively. After the MCPs, the electron cloud is attracted by the delay-line anode. The induced signal propagates to both ends of the delay-line wire [cf. Fig. 4(c)]. One of the position coordinates is given by $x=v_{\perp} / 2\left(t_{2}-t_{1}\right)$. Here, $t_{1}=t_{1}^{*}-t_{\mathrm{MCP}}$ and $t_{2}=t_{2}^{*}-t_{\mathrm{MCP}}$ are the arrival times of the signal at the ends of the wire relative to the MCP timing signal $t_{\mathrm{MCP}}$. The effective propagation velocity of the signal along the wire is denoted by $v_{\perp}$.

The ion detector is equipped with a stack of two $120 \mathrm{~mm}$ diameter MCPs in a chevron arrangement [cf. Fig. 4(b)] and a delay-line anode. Specifically, it is a so-called quadanode as it consists of two perpendicularly oriented quadratic wire layers [cf. Fig. 4(a)]. The electron detector features three MCPs of $80 \mathrm{~mm}$ diameter in a $Z$-arrangement and a delay-line anode, which is made of three wire layers that are rotated by $60^{\circ}$

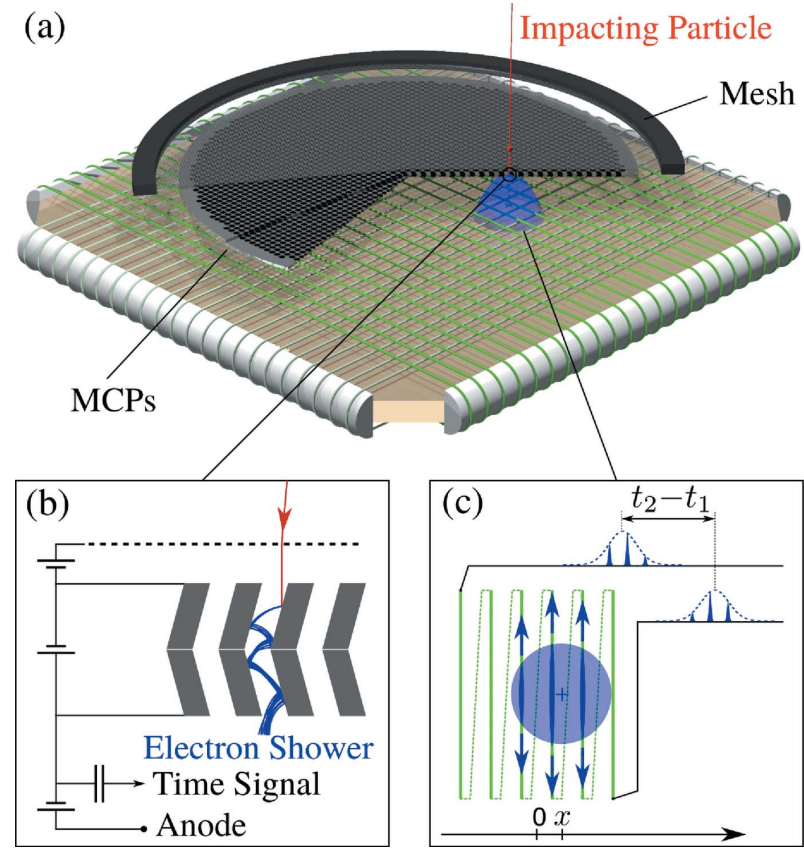

Figure 4

(a) CAD-like drawing showing the working principle of the time- and position-sensitive detector. (b) Amplification by the MCPs and outcoupling of the TOF signal. (c) Position detection scheme using a delayline anode. The figure is adapted from Fechner (2014). [Reprinted by permission from Springer.]

relative to each other. This so-called hexanode delay-line anode improves the multi-particle hit detection efficiency by introducing redundancy (Jagutzki et al., 2002a).

\subsection{Vacuum conditions}

All ions and electrons, which are created inside the volume of the spectrometer ( $c f$. Section 2.2), are accelerated towards the detectors ( $c f$. Section 2.3) and are registered as events. The detectors, however, cannot discriminate between particles from the target and those from residual gas. At XUV photon energies above $\sim 15 \mathrm{eV}$, a single photon can ionize a residual gas molecule (e.g. $\left.\mathrm{H}_{2}, \mathrm{H}_{2} \mathrm{O}, \mathrm{N}_{2}, \mathrm{O}_{2}\right)$. The XUV photoabsorption cross sections of residual-gas molecules and those of target atoms, molecules or clusters are of the same order of magnitude (Yeh \& Lindau, 1985; Gallagher et al., 1988). In combination with FEL pulse energies of the order of some tens of $\mu \mathrm{J}$, this causes a non-negligible number of background events along the FEL beam inside the REMI spectrometer.

To minimize the number of background events, vacuum conditions of $\leq 10^{-11}$ mbar need to be reached in the REMI interaction chamber. The generation of such high vacua in a large vessel like the REMI interaction chamber (CF DN250 tube, length $\sim 80 \mathrm{~cm}$ ) is difficult to achieve by turbomolecular pumps alone. Thus, the complete inner surface of the interaction chamber is coated with a thin film $(\sim 1.5 \mu \mathrm{m})$ of nonevaporable getter (NEG) material. NEG is a special alloy, e.g. $\mathrm{Ti}-\mathrm{Zr}-\mathrm{V}$, which has a large surface area. Thereby, the probability of molecules to stick to the chamber walls is increased. At extreme vacua $\left(\sim 10^{-12}\right.$ mbar $)$, the thermal 
outgassing of $\mathrm{H}_{2}$ from the steel walls of the chamber is the major source of residual gas (Benvenuti et al., 1999). However, turbomolecular pumps are not well suited to pump $\mathrm{H}_{2}$ with typical $\mathrm{H}_{2}$ pumping speeds of only several hundreds $1 \mathrm{~s}^{-1}$ (Jousten, 2010). In contrast, the $\mathrm{H}_{2}$ pumping speed of the NEG coating of the REMI interaction chamber is estimated to be $\sim 3600 \mathrm{l} \mathrm{s}^{-1}$ (Benvenuti et al., 1999). However, after each venting, the coating needs to be re-activated by heating the chamber to temperatures of $\geq 200^{\circ} \mathrm{C}$ for at least $24 \mathrm{~h}$ (Benvenuti et al., 1999).

\subsection{Data acquisition}

FLASH features a special pulse repetition rate pattern. At $10 \mathrm{~Hz}$ repetition rate, pulse trains (maximum train length $800 \mu$ s) with an intra-train pulse separation between 1 and $25 \mu$ s are provided (Faatz et al., 2016). This burst-mode pulse pattern requires a data acquisition system, which is capable of processing the incoming signals of a REMI.

Four Acqiris DC282 digitizer cards, each with four channels, are used to record the voltage traces of each and every detector signal over a time range up to $800 \mu$ s. The highest sampling rate of the digitizer cards is $2 \mathrm{GHz}(0.5 \mathrm{~ns}$ between two samples) and the maximum voltage range for each channel is $\pm 5 \mathrm{~V}$. The voltage trace for each channel recorded during the entire pulse train is stored internally on the digitizer cards. Then, the $10 \mathrm{~Hz}$ FEL trigger initiates the readout of the channels after the end of each pulse train. Zero suppression, peak finding on the signals and sorting of the data is done by computer algorithms in the online as well as the offline analysis (Schnorr, 2014). The analysis code GENERiC (General Analysis Code for Reaction Microscopes) is embedded in the Go4 (GSI Object Oriented On-line Off-line system) analysis environment (GSI, 2018) based on CERN's ROOT (CERN, 2018).

\section{In-line XUV split-delay and focusing optics}

The basic concept of the XUV optics is to split and focus the FEL beam before it enters the REMI.

The underlying geometry and working principle is depicted in Fig. 5. The FEL beam approaches from the left and first hits the split-mirror under a grazing angle of $\theta=8^{\circ}$. The splitmirror is a plane mirror, which is horizontally cut into two coextensive pieces. The upper mirror is movable and can be translated back and forth along the mirror normal axis. Thus, a path difference can be introduced between the upper beam (green) and the lower beam (blue). The incoming FEL pulse is split into two and the path difference translates into a temporal delay $t_{\mathrm{d}}$ between the pump (blue) and the probe (green) pulse. After the splitting, the two beams are focused by an ellipsoidal mirror into the supersonic jet of the REMI. The grazing-incidence geometry and a carbon coating of the split and the ellipsoidal mirror guarantee high reflectivity over a broad photon energy range from 30 to $180 \mathrm{eV}$ ( $c f$. Fig. 7). This allows making use of the wavelength tunability of the FLASH2 variable-gap undulators (Faatz et al., 2016). The use

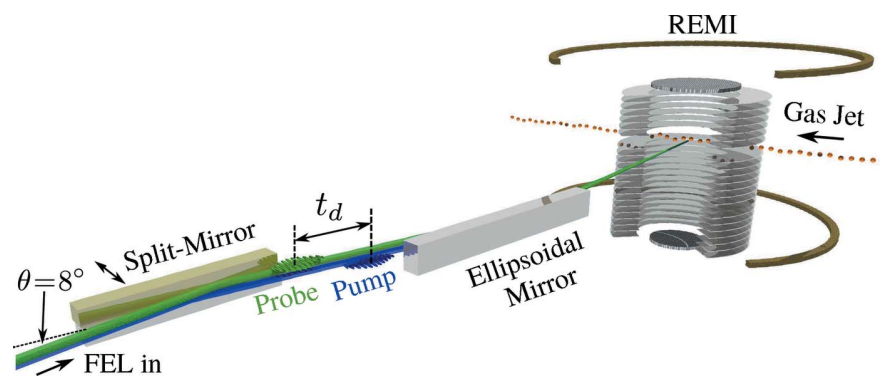

Figure 5

Design of the in-line XUV split-delay and focusing optics. Shown are the FEL beam path, the split-mirror, the ellipsoidal mirror and the REMI with gas jet. Also depicted is the generation of a pump (blue) and a probe (green) pulse by translating the upper split-mirror along the mirror normal axis. Pump and probe pulses are temporally delayed by $t_{\mathrm{d}}$. The figure is adapted from Schmid (2018).

of a single ellipsoidal mirror instead of a KB focusing system, implemented at other beamlines at FLASH (Erk et al., 2018), reduces the number of optical components in the beam path and thus increases the overall transmission of the beamline.

\subsection{Split-mirror assembly}

The split-mirror is the centerpiece of the XUV pump-probe optics. It consists of two plane mirrors with clear apertures of $120 \mathrm{~mm} \times 10.5 \mathrm{~mm}$ each. In order to achieve femtosecond pump-probe resolution and to keep spatial overlap of both beams in the focus for all delay values, the upper mirror is movable by a kinematics based on piezo positioners. The splitmirror assembly is shown in Fig. 6.

The kinematics is custom-made by SmarAct GmbH. It allows the position of the upper mirror to be manipulated in three dimensions: linear motion along the mirror normal axis (travel), rotation along the vertical (tilt) and horizontal (tip) axis. The coordinate system is defined in Fig. 6. The rotational

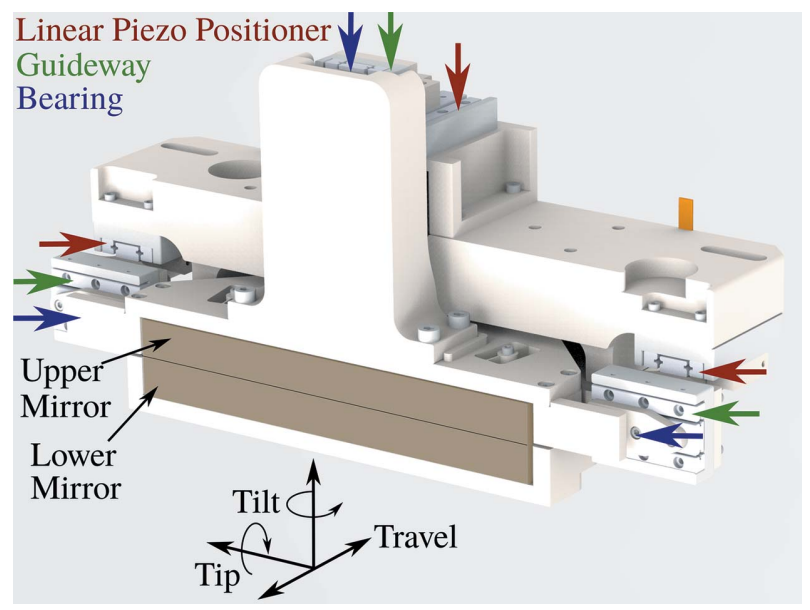

Figure 6

Split-mirror assembly. The upper mirror is movable in the three dimensions travel, tilt and tip by a piezo-driven kinematics. Positioners, guideways and bearings are marked by red, green and blue arrows, respectively. Details on the assembly are given in the main text. The figure is adapted from Schmid (2018). 
axes are set along the mirror surface. The maximum travel range of $h= \pm 3 \mathrm{~mm}$ along the mirror normal translates into a maximum delay range of $t_{\mathrm{d}}= \pm 2.7 \mathrm{ps}$. Rotations up to a few degrees are possible for the tilt axis. The maximum tip rotation of $\pm 20 \mathrm{mrad}$ is limited by the minimal allowed gap distance of $100 \mu \mathrm{m}$ between the two mirrors. The gap is intended to be as small as possible in order to minimize photon losses. The distance between the mirrors can be manually adjusted by three spring-mounted fine-thread screws.

The kinematics has three legs, each consisting of a linear piezo positioner, a linear guideway and a bearing ( $c f$. Fig. 6). Due to the guideways and the bearings, linear motions of the piezo positioners translate into a linear motion along the travel direction and rotations around the tip or tilt axis. The positioners are SmarAct SLC-1730 nanometer-precision linear piezo positioners, which are based on the stick-slip principle (Hunstig, 2017). They are operated in closed-looped (encoder feedback) and are controlled by the SmarAct MCS controller module with the Advanced Sensor Calibration (ASC) option.

Based on the position resolution of the encoders $(4 \mathrm{~nm})$, the upper limit of the temporal resolution is estimated to be $\Delta t_{\mathrm{d}} \simeq 4$ as. Tests employing the interference pattern of an optical laser (wavelength $658 \mathrm{~nm}$ ) confirm this limit. Tests on the pointing stability, i.e. the ability to maintain spatial overlap of both beams in the focus for all delays, are presented in Section 5.2.

\subsection{Ellipsoidal mirror}

After the split-mirror, the beam is focused by an ellipsoidal mirror (cf. Fig. 5). It is designed for a source distance of $85 \mathrm{~m}$, a focal length of $1 \mathrm{~m}$ and an incidence angle of $\theta=8^{\circ}$. The machining of the mono-crystalline silicon substrate was performed by Carl Zeiss SMT GmbH. The clear aperture of the mirror is $180 \mathrm{~mm} \times 20 \mathrm{~mm}$. The quality inspection of the manufacturer specifies the slope errors to be 0.84 arcsec (r.m.s.) tangential and $1.2 \operatorname{arcsec}$ (r.m.s.) sagittal. The surface roughness is measured to be $0.19-0.24 \mathrm{~nm}$. For high transmission over a broad XUV photon energy range, the mirror is coated with a $30 \mathrm{~nm}$ thin layer of carbon by optiXfab GmbH. The layer thickness has a relative error of $\pm 5 \%$ and the lateral homogeneity is $>97 \%$. At $\theta=8^{\circ}$, the mirror has a reflectivity of $>50 \%$ up to photon energies of $180 \mathrm{eV}$ ( $c f$. Fig. 7).

The split-mirrors are also carbon-coated in the same way and the mono-crystalline silicon substrates are of the same quality as the ellipsoidal mirror.

\subsection{Hexapod kinematics}

To obtain best focusing conditions and high transmission, the split and the ellipsoidal mirror need to be individually positioned in all three rotational and translational directions with microradian- and micrometre-precision. In order to achieve these accuracies, hexapod kinematics as shown in Fig. 8 are used.

The split-mirror assembly and the ellipsoidal mirror have their individual kinematics, which are mounted on two separate flanges of one vacuum chamber. A decoupled adjustment

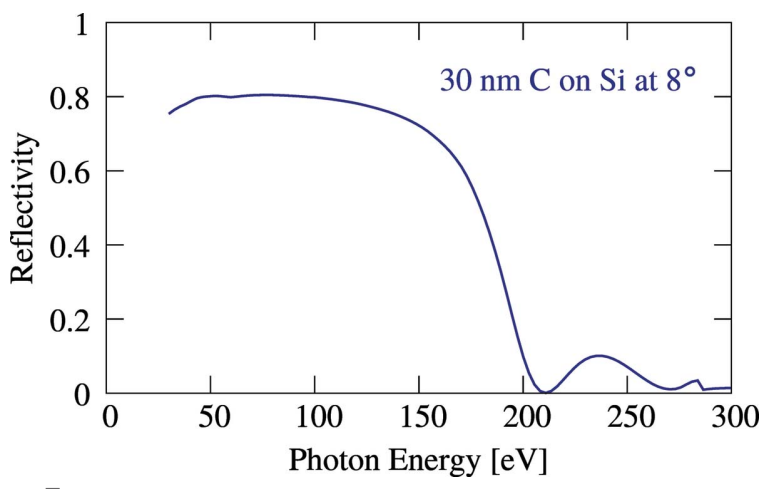

Figure 7

Calculated reflectivity at a grazing angle of $\theta=8^{\circ}$ of a carbon-coated mirror (30 nm layer thickness, mono-crystalline silicon substrate). Data taken from Henke et al. (1993).

of both mirrors is essential for beam alignment and also indispensable to achieve optimal focusing conditions as well as to compensate manufacturing tolerances of the mirror chamber. The mounting on two separate supporting flanges also enables an independent removal of both mirror assemblies.

The general design of the kinematics has been developed by the group around T. Noll and Th. Zeschke from the Helmholtz Zentrum Berlin (HZB)/BESSYII (Noll et al., 2009). The design has been adapted by FMB Berlin $\mathrm{GmbH}$, who also manufactured the kinematics and the surrounding vacuum chamber.

The hexapod has six legs, each consisting of two steel rope joints, a connection strut, a leaf spring, a bellow and a motorized actuator ( $c f$. Fig. 8). By linearly pulling or pushing of the actuators, the legs react dynamically and the attached platform moves. The design uses stable steel rope joints instead of conventional bearings to increase the overall stiffness. The flexible copper-beryllium leaf springs prevent a lateral displacement of the upper joint in stressed positions of the joints. In contrast to usual hexapods, the driving motors are not directly part of the moving assembly. This decreases the total mass and potentially outgassing electrical parts stay

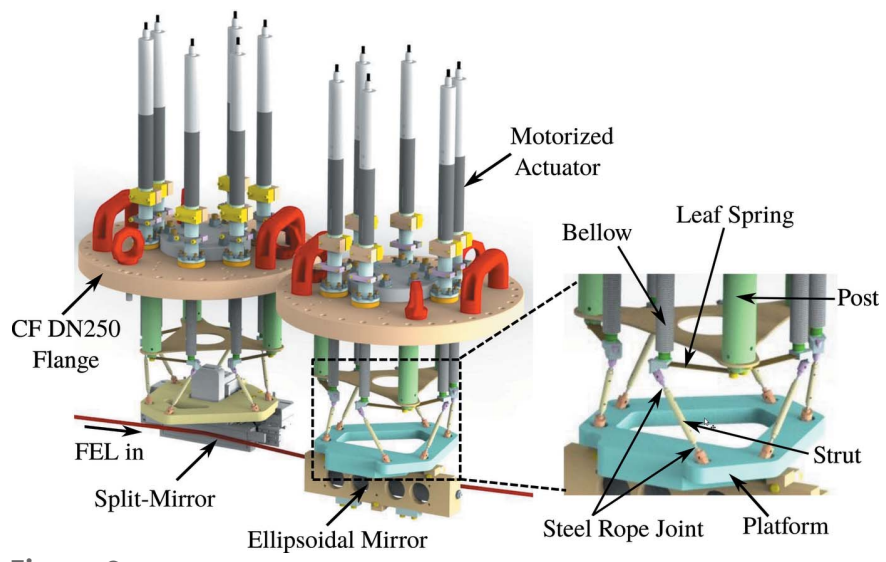

Figure 8

CAD-like drawing of the two hexapod kinematics holding the splitmirror assembly and the ellipsoidal mirror, respectively. Labels mark the main parts. The figure is adapted from Schmid (2018). 
outside the vacuum. Bellows separate the actuators from the vacuum. After baking of the vacuum chamber at $120^{\circ} \mathrm{C}$ for about $24 \mathrm{~h}$, a pressure of $10^{-10} \mathrm{mbar}$ was reached in the mirror chamber.

The hexapods are designed for rotations of $R_{x}, R_{y}, R_{z}= \pm 0.5^{\circ}$ and translations of $T_{x}, T_{y}= \pm 3 \mathrm{~mm}$ laterally and $T_{z}= \pm 5 \mathrm{~mm}$ vertically. The vertical travel of $T_{z}= \pm 5 \mathrm{~mm}$ for the splitmirror assembly allows the complete FEL beam (10 $\mathrm{mm}$ diameter) to be put onto one of the split-mirrors. This option is used for experiments, which do not employ an XUV-XUV pumpprobe scheme, e.g. XUV-IR pumpprobe experiments ( $c f$. Section 6). Tests have shown that the resolution for all translations is $\Delta T_{x, y, z} \simeq 0.1 \mu \mathrm{m}$ and for all rotations $\Delta R_{x, y, z} \simeq 1 \mu \mathrm{rad}$. The movement of the hexapods is controlled by a LabVIEW program. ${ }^{2}$

\section{Beamline}

\subsection{Accelerator tunnel section and} experimental hall

An overview of the FEL beam transport and diagnostics at FLASH2 has been given by Plönjes et al. (2016). In the tunnel section, beam position monitors and a set of apertures followed by Ce:YAG fluorescence screens are used to monitor and to shape the FEL beam. The apertures are also utilized to adjust the transmitted pulse energy. The absolute pulse energy can be measured by a gas-monitor detector (GMD) (Tiedtke et al., 2009) or a micro-channel plate (MCP) tool (Bittner $e t$ al., 2007). A non-invasive online photoionization spectrometer (OPIS) measures the average photon energy (Braune $e t$ al., 2016). The accelerator tunnel section ends with a gas-filled attenuator, which allows the transmitted energy per pulse to be continuously adjusted.

The photon-diagnostics section in the experimental hall is equipped again with a second GMD, a further set of apertures and Ce:YAG fluorescence screens. Transmission filters are mounted to regulate the FEL intensity and to suppress harmonics. The filters of different materials and thicknesses (typically a few $100 \mathrm{~nm}$ ) are mounted on two consecutive motorized wheels. Depending on the photon energy and intensity, different filters and combinations can be selected.

\subsection{REMI beamline}

In order to protect the vacuum conditions in the REMI interaction chamber $\left(\leq 10^{-11} \mathrm{mbar}\right)$ from the vacuum in the FLASH beamline (typically $>10^{-9}$ mbar) and to reduce stray

\footnotetext{
${ }^{2}$ The core part of the program has been gratefully adopted from J. P. Müller from the TU Berlin used for the KB optics at the CAMP endstation at FLASH1.
}

light, the beamline in front of the REMI is separated into five differentially pumped sections and has a total length of $\sim 6.3 \mathrm{~m}$ ( $c f$. Fig. 9).

The sections are connected via small-diameter tubes (colored orange in Fig. 9) which leads to low gas conductance between the sections. The tubes are configured to reduce stray light. At the entrance and exit, chamfered copper apertures are clamped into the tubes. The chamfer is manufactured as sharp as possible in order to minimize the area where photons might scatter. The apertures have an inner diameter of $18 \mathrm{~mm}$, while the tubes (lengths between 185 and $287 \mathrm{~mm}$ ) themselves have an inner diameter of $26 \mathrm{~mm}$ [cf. Fig. 9(a)]. This configuration acts as a light baffle for photons that are scattered in off-axis directions.

After the mirror chamber, a further light baffle (colored blue in Fig. 9) is installed to prevent stray light in the direct vicinity of the REMI detectors. This light baffle consists of two apertures with respective inner diameters of $14 \mathrm{~mm}$ and $12 \mathrm{~mm}$ [cf. Fig. 9(b)]. They are made of roughened $625 \mu \mathrm{m}$ thin silicon wafers and serve the same purpose as the copper apertures of the differential tubes, i.e. to remove undirected scattered photons.

At two distinct positions along the beamline, circular apertures on a moveable rail (aperture diameters from 4 to $10 \mathrm{~mm}$ in steps of $1 \mathrm{~mm}$ ) are integrated (colored dark-green in Fig. 9). The apertures are chamfered with the sharp edge pointing towards the incoming beam again for reasons of stray light reduction. Additionally, the apertures' surfaces facing the beam are coated with a fluorescent $\mathrm{ZnS}$ powder, which facil- 
itates the beam alignment. A third aperture (diameter $10 \mathrm{~mm}$ ) which is part of the IR incoupling unit (colored red in Fig. 9, discussed in Section 6) is located directly in front of the REMI interaction chamber.

In total, three fluorescence screens (colored light-green in Fig. 9) can be inserted to monitor the position and the shape of the FEL beam. The first screen $(20 \mathrm{~mm} \times 20 \mathrm{~mm})$ is located in front of the mirror chamber. A second screen $(25 \mathrm{~mm} \times 25 \mathrm{~mm})$ can be placed between the split and the ellipsoidal mirror to monitor the shape of the split beam and the illumination uniformity on the split-mirrors. A third screen $(20 \mathrm{~mm} \times 20 \mathrm{~mm})$ is located in front of the REMI. Each screen consists of a stack of a Ce:YAG plate $(200 \mu \mathrm{m}$ thick $)$ and a roughened silicon plate $(625 \mu \mathrm{m}$ thick $)$. The Ce:YAG plate faces the FEL beam and fluoresces upon illumination with XUV photons. The roughened silicon plate, which is mounted behind the Ce:YAG plate, is used to visualize the optical alignment laser (wavelength $658 \mathrm{~nm}$ ).

A pair of horizontal slits (colored light-brown in Fig. 9) can be used to block the upper or the lower part of the incoming FEL beam. This option is useful for adjusting the downstream XUV split-delay and focusing optics (cf. Section 3), e.g. to check how the pulse energy in the pump and the probe pulse is distributed.

\subsection{REMI interaction chamber and photon dump}

In order to spatially overlap the foci at the nominal interaction point with the gas jet ( $c f$. Section 3), a fluorescence screen can be moved into the center of the REMI spectrometer. This target screen is $10 \mathrm{~mm} \times 10 \mathrm{~mm}$ in size and coated with a thin film of Ce:YAG powder. A CCD camera (Basler acA1300-30gc with Sony ICX445 CCD sensor, 1.3 MP, pixel size $3.75 \mu \mathrm{m} \times 3.75 \mu \mathrm{m}$, sensor size $4.9 \mathrm{~mm} \times 3.6 \mathrm{~mm}$ ) in combination with an optical magnification system (Navitar 1-60135D, overall magnification 2.4) images the fluorescence of the foci from outside. The vacuum of the REMI interaction chamber is separated from the following photon dump by two differentially pumped sections. The corresponding differential tubes are colored orange in Fig. 10.

The beam profile and position after the REMI can be monitored by a Ce:YAG fluorescence screen $(20 \mathrm{~mm} \times$ $20 \mathrm{~mm}$ ) (colored light-green in Fig. 10). The fluorescence screen is followed by a $1 \mathrm{~m}$-long CF DN40 tube. Two bellows (colored yellow in Fig. 10) at each end allow to slightly tilt or bank out the entire tube. This option is applied to minimize photon back-scattering from the FEL dump. The photon dump is terminated by a copper plate, which is electrically connected (colored brown in Fig. 10). The voltage signals induced by impinging FEL pulses serve as a reference for the pulse arrival times and the pulse energies. From the first valve (colored light-blue in Fig. 10) onwards, the entire photon dump is mounted on a single supporting frame. This way, it can be quickly removed for setting up a subsequent optional experiment behind the REMI. The first valve is located $827 \mathrm{~mm}$ behind the center of the REMI. Taking the maximum diameter of $10 \mathrm{~mm}$ of the incoming FEL beam before focusing, the diverging FEL beam has a maximum diameter of $\sim 8.3 \mathrm{~mm}$ at this position.

\section{Commissioning results}

\subsection{Focal spot size}

A wavefront sensor (WFS) (Keitel et al., 2016) has been used to analyze the optical properties of the new XUV splitdelay and focusing optics ( $c f$. Section 3). For the measurements at $h \omega=56 \mathrm{eV}$ a Compact Hartmann Sensor (Keitel et al., 2016) was used. The intensity profile of the smallest achieved focus is shown in Fig. 11.

A diameter of $d \simeq 3 \mu \mathrm{m}$ (FWHM) was obtained. Assuming a pulse energy of $E_{\text {pulse }}=10 \mu \mathrm{J}$ and a typical pulse duration of $T=50 \mathrm{fs}$, this corresponds to an intensity of $I \simeq 10^{15} \mathrm{~W} \mathrm{~cm}^{-2}$.

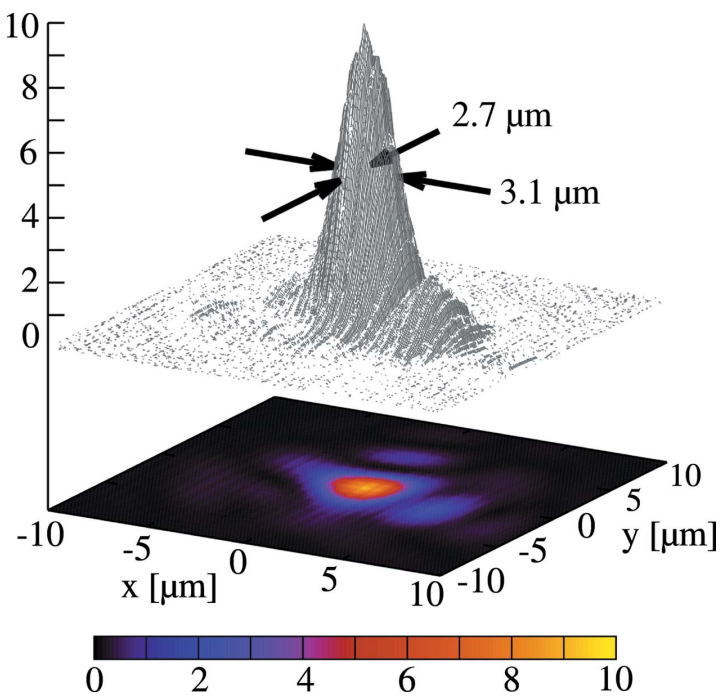

Figure 11

Intensity profile obtained by wavefront reconstruction. Shown is the smallest achieved focus. The arrows mark the FWHM in the $x$ - and $y$ direction. 


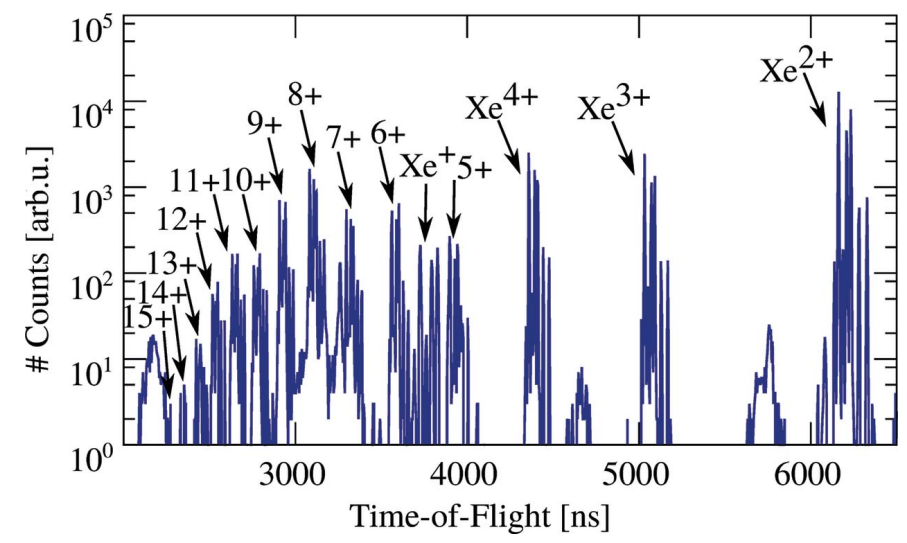

Figure 12

TOF spectrum of xenon ions recorded at $h \omega=100 \mathrm{eV}$. For this measurement, the entire FEL beam with $E_{\text {pulse }} \simeq 20 \mu \mathrm{J}$ pulse energy is put onto the lower mirror of the split-mirrors. $\mathrm{Xe}^{(1-15)+}$ ions are marked by arrows. As TOF $\propto(m / q)^{1 / 2}\left[c f\right.$. equation (1)], $\mathrm{Xe}^{+}$ions have the longest TOFs of all $\mathrm{Xe}$ ions considered, which is longer than the time interval $\Delta t_{\mathrm{FEL}}=5000 \mathrm{~ns}$ between two consecutive FEL pulses. Therefore, $\mathrm{Xe}^{+}$ ions appear out of order at $\mathrm{TOF}\left(\mathrm{Xe}^{+}\right)-\Delta t_{\mathrm{FEL}}=3750 \mathrm{~ns}$. The figure is taken from Schmid (2018).

The charge states reached in multi-photon ionization of xenon are a further benchmark of the achieved intensity and thus the focus size. Fig. 12 shows the TOF spectrum of xenon ions recorded at a photon energy of $\hbar \omega=100 \mathrm{eV}$ and a pulse energy of $E_{\text {pulse }} \simeq 20 \mu \mathrm{J}$.

For this measurement, the entire FEL beam was placed onto the lower split-mirror to avoid losses due to the gap between the two mirrors. For each charge state several closelying TOF peaks appear reflecting the naturally abundant isotopes of xenon. The charge states are marked by arrows and $\mathrm{Xe}^{15+}$ is the highest charge state observed. Richter et al. (2009) measured $\mathrm{Xe}^{14+}$ at $I=1.7 \times 10^{15} \mathrm{~W} \mathrm{~cm}^{-2}$ and Sorokin et al. (2007a) reported $\mathrm{Xe}^{15+}$ at $I=2 \times 10^{15} \mathrm{~W} \mathrm{~cm}^{-2}$. Taking these values, the intensity reached with the present setup is estimated to be $1 \times 10^{15}$ to $2 \times 10^{15} \mathrm{~W} \mathrm{~cm}^{-2}$. This is in accordance with the focal spot size measured with the WFS for the given pulse energy of $E_{\text {pulse }} \simeq 20 \mu \mathrm{J}$ and an estimated pulse duration of $T=50 \mathrm{fs}$. In XUV pump-probe operation, the FEL beam is put on both split mirrors. In this configuration, the highest recorded charge state is $\mathrm{Xe}^{11+}$, which according to Sorokin et al. (2007a) corresponds to an intensity of $I \simeq 5 \times 10^{14} \mathrm{~W} \mathrm{~cm}^{-2}$.

\subsection{Pointing stability of the split-mirrors}

It is crucial for any pump-probe experiment to maintain spatial overlap of the two foci during delay scans. In order to determine the pointing stability of the split-mirror assembly (cf. Section 3.1), the target screen is moved to the nominal focal position in the center of the REMI spectrometer (cf. Section 4.3). The FEL intensity needs to be strongly attenuated by the beamline filters ( $c f$. Section 4.1) to not destroy the screen. The fluorescence spots of the movable and the fixed focus are then observed by the CCD camera with the optical magnification system from outside ( $c f$. Section 4.3).
The CCD camera is read out with the $10 \mathrm{~Hz}$ FEL trigger. The two foci are spatially separated by about $150 \mu \mathrm{m}$, which allows the centroid positions of the fluorescence spots [spot sizes $64 \pm 12 \mu \mathrm{m}$ (FWHM)] of the movable and the fixed focus to be determined. To this end, both spots are selected by regions of interest. These regions are then projected, background is subtracted and the centroids are calculated from the center-ofmass of the projections. A sub-pixel resolution of below $1.6 \mu \mathrm{m}$ is reached with this method. The delay is scanned and for each delay step the centroid position of the spot of the movable focus is determined relative to that of the fixed focus in the horizontal $(x)$ and vertical $(y)$ direction. The relative deviation $\Delta x$ of the centroid positions in the $x$-direction is plotted as a function of the pump-probe delay in Fig. 13(a).

For this plot, the entire delay range has been scanned four times. Between $\Delta x(-2000 \mathrm{fs})=+15 \mu \mathrm{m}$ and $\Delta x(+2000 \mathrm{fs})=$ $-30 \mu \mathrm{m}$, an absolute deviation of $45 \mu \mathrm{m}$ exists. Additionally, anharmonic oscillations are superimposed on this almost linear slope. The origin of this non-linear behavior is not understood. It might be caused by an imperfect synchronization of the movements of the three piezo positioners (cf. Section 3.1). In order to compensate the slope and the anharmonic oscillations, the position versus delay curve is recorded in a separate calibration measurement and interpolated by splines, which is then used for correcting the movements of the piezo kinematics. Four delay scans using the correction are superimposed in Fig. 13(b). The small width of the distribution [cf. inset of Fig. 13(b)] shows the high precision and reproducibility of the piezo kinematics. The $1 \sigma$ deviation over the entire delay range is $\sigma_{\Delta x}=(2.2 \pm 0.5) \mu \mathrm{m}$. In the vertical direction $\Delta y$, corrections are also necessary and a comparable stability is achieved as well. Hence, for pumpprobe experiments, the two foci are spatially well overlapped when the delay-dependent correction obtained by the method introduced above is applied.

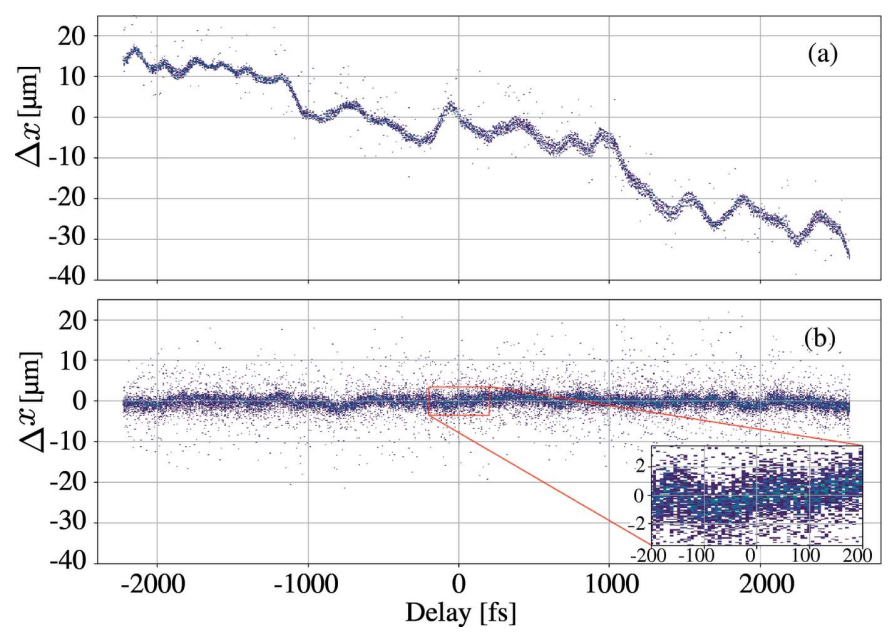

Figure 13

Relative deviation $\Delta x$ of the centroid positions of the fluorescence spots of the movable and the fixed focus on the target screen as a function of the pump-probe delay. (a) Without correction. (b) With correction. The inset shows a zoom-in between $-200 \mathrm{fs}$ and +200 fs. For both plots, the delay range is scanned four times. 


\subsection{Stray-light reduction}

Stray light, i.e. scattered XUV photons that trigger events at the ion or electron detector of the REMI (cf. Section 2.3), is a major source of background events. With the in-line XUV split-delay and focusing optics ( $c f$. Section 3), a significant reduction of stray light is achieved compared with a formerly used back-reflecting split-mirror (Jiang et al., 2010a; Schnorr et al., 2013). Using similar FEL pulse energies, the stray light background is reduced by a factor of about 15 . In the previous setup, an FEL beam of $10 \mathrm{~mm}$ diameter first passed the REMI interaction chamber before it was focused into the gas jet. This back-reflecting geometry facilitated undirected scattered photons. With the new split-delay and focusing optics, however, a well collimated FEL beam passes the REMI interaction chamber only once.

\subsection{Electron-ion coincidences}

As a next step, after commissioning of the FEL beamline, the REMI itself was tested. To this end, two-photon doubleionization (TPDI) of neon at a photon energy $\hbar \omega=44 \mathrm{eV}$ has been studied using the in-line XUV optics ( $c f$. Section 3). At a photon energy of $44 \mathrm{eV}$, TPDI proceeds via the so-called sequential channel: the absorption of one photon leads to the formation of a real intermediate state of the singly charged $\mathrm{Ne}^{+}$ion, which then absorbs a second photon resulting in a doubly charged $\mathrm{Ne}^{2+}$ ion,

First step: $\quad \mathrm{Ne}+\hbar \omega(44 \mathrm{eV}) \longrightarrow \mathrm{Ne}^{+}+\mathrm{e}_{1}$,

Second step: $\quad \mathrm{Ne}^{+}+\hbar \omega(44 \mathrm{eV}) \longrightarrow \mathrm{Ne}^{2+}+\mathrm{e}_{2}$.

Although the two ionization steps might be regarded as completely independent at first glance, the two ejected electrons $\left(\mathrm{e}_{1}\right.$ and $\left.\mathrm{e}_{2}\right)$ are correlated, which is, for example, reflected in the angular distributions of the first- and secondstep electrons (Kheifets, 2007; Fritzsche et al., 2008; GrumGrzhimailo et al., 2009). To unambiguously measure the angular distribution of the first-step electron in TPDI, this electron needs to be distinguished from an electron emerging from another ionization event. This is possible, if the two ejected electrons $\left(\mathrm{e}_{1}\right.$ and $\left.\mathrm{e}_{2}\right)$ and the corresponding $\mathrm{Ne}^{2+}$ ion are measured in coincidence [ $c f$. equation (2)].

A so-called photoelectron-photoelectron-photoion coincidence (PePePiCo) map may be used as feedback as to whether the coincident detection of electrons and ions was successful. In such a plot, the ion TOF is plotted against the sum of the TOFs of the first and the second electron arriving at the detector. The PePePiCo plot for TPDI of neon at a photon energy of $\hbar \omega=44 \mathrm{eV}$ is shown in Fig. 14.

In a true coincident event, the sum momentum of all ejected particles (electrons and ions) is constant. As the TOF of a particle is in first approximation directly proportional to its momentum, the diagonal line in Fig. 14 indicates the coincident detection of a $\mathrm{Ne}^{2+}$ ion and the two ejected electrons. According to the geometry of the REMI spectrometer (cf. Fig. 2), an ion that starts with an initial momentum towards the ion detector has a shorter TOF. Due to momentum conservation, the corresponding electrons start with initial

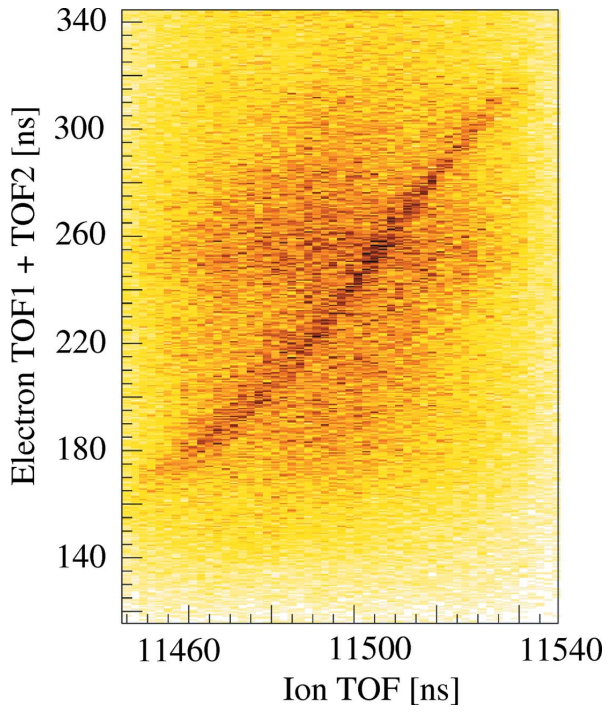

Figure 14

Photoelectron-photoelectron-photoion coincidence (PePePiCo) map for two-photon double ionization of neon at $\hbar \omega=44 \mathrm{eV}$. The ion TOF is plotted on the horizontal axis. The vertical axis shows the sum of the TOFs of the first and second detected electron impinging on the detector. In total, $1.6 \times 10^{7} \mathrm{FEL}$ pulses were taken to produce the histogram. The figure is taken from Schmid (2018).

momenta towards their detector and the sum TOF1 + TOF2 is short as well. In the opposite case, i.e. where the ion has an initial momentum towards the electron detector, the ion TOF is larger and thus also the sum TOF of the two electrons is larger. The PePePiCo map of Fig. 14 shows that with the background reduction ( $c f$. Section 5.3) achieved by the design of the beamline ( $c f$. Section 4.2) and by focusing with the inline XUV optics ( $c f$. Section 3), detailed studies on two- and multi-photon ionization of atoms, molecules and small clusters are possible at the REMI endstation. In contrast to previous experiments, e.g. TPDI of $\mathrm{Ne}$ at $44 \mathrm{eV}$ has already been studied using a REMI at FLASH1 (Kurka et al., 2009), these studies can now be carried out in a time-resolved manner using the in-line XUV split-delay and focusing optics ( $c f$. Section 3).

\subsection{Delay-dependent kinetic energy release spectra}

The fragmentation of argon dimers $\left(\mathrm{Ar}_{2}\right)$ at a photon energy of $\hbar \omega=24.8 \mathrm{eV}$ is taken as a case study to test the pump-probe capability of the split-delay and focusing optics. The kinetic energy release (KER) of the dimer fragments is measured as a function of the pump-probe delay. The KER is approximately given by

$$
\mathrm{KER} \simeq \frac{q_{1} q_{2}}{R}
$$

with $q_{1}$ and $q_{2}$ being the ion charges [atomic units are used in equation (3)]. The dependence of the KER on the internuclear distance $R$ allows conclusions to be drawn on the nuclear dynamics during the fragmentation. In Fig. 15, the KER of the $\mathrm{Ar}^{+}+\mathrm{Ar}^{+}$coincidence channel is plotted as a function of the pump-probe delay $t_{\mathrm{d}}$. 


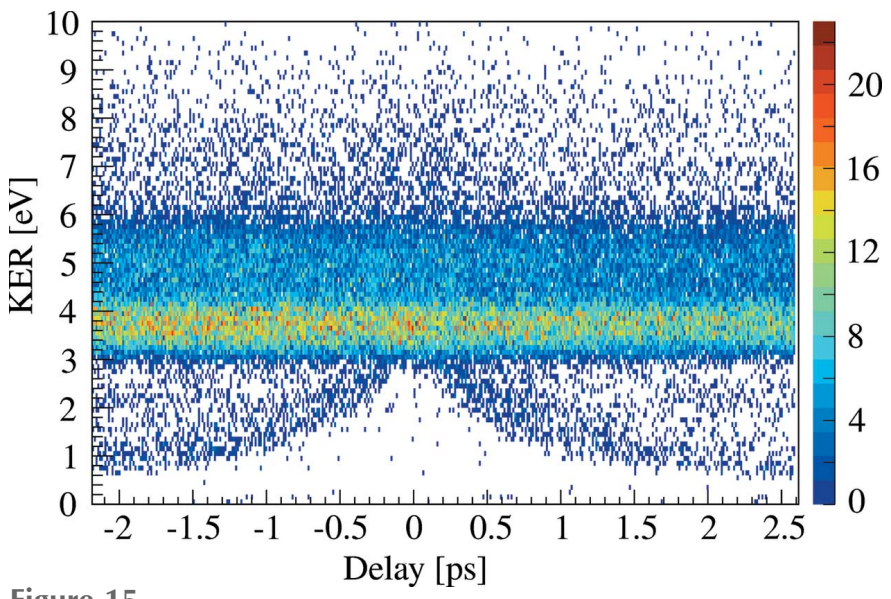

Figure 15

KER of $\mathrm{Ar}^{+}+\mathrm{Ar}^{+}$ions as a function of the pump-probe delay. The data are recorded at a photon energy of $\hbar \omega=24.8 \mathrm{eV}$ and combined FEL intensities of $I_{\mathrm{FEL}}=10^{13}-10^{14} \mathrm{~W} \mathrm{~cm}^{-2}$. The pulse energies in the pump and probe are approximately equal. The figure is taken from Schmid (2018).

Over the entire range, delay-independent contributions at $3.8 \mathrm{eV}$ and $5.3 \mathrm{eV}$ are present. They stem from the absorption of two photons within one pulse, the pump or the probe. The contribution at $3.8 \mathrm{eV}$ is assigned to the direct fragmentation at the equilibrium internuclear distance $R_{\mathrm{eq}}=7.18$ a.u. by absorbing two photons. As depicted by the potential energy curves (PECs) in Fig. 16, this happens sequentially via the intermediate $\operatorname{Ar}^{+}\left(3 p^{-1}\right)-\operatorname{Ar}$ state within one FEL pulse. The contribution at $5.3 \mathrm{eV}$ is induced by an interatomic Coulombic decay (ICD) of a one-site ionized and excited $\operatorname{Ar}^{+}\left(3 p^{-2} n l\right)-\mathrm{Ar}$ state (Miteva et al., 2014) (cf. Fig. 16). The decay onto the repulsive $\operatorname{Ar}^{+}\left(3 p^{-1}\right)-\operatorname{Ar}^{+}\left(3 p^{-1}\right)$ state takes place at an internuclear distance $R<R_{\mathrm{eq}}$, which according to equation (3) results in a higher KER compared with the fragmentation at $R_{\text {eq. }}$.

Besides the delay-independent contributions, a pumpprobe signal is visible in Fig. 15. It starts at time zero at $3.8 \mathrm{eV}$ and asymptotically reaches KERs of $\sim 1.0 \mathrm{eV}$ for large delays. This signal is attributed to dissociative photoionization. The pump pulse initiates the dissociation of the dimer into a charged and a neutral fragment ( $c f$. Fig. 16),

$$
\text { Pump: } \mathrm{Ar}-\mathrm{Ar} \stackrel{\mathrm{XUV}}{\longrightarrow} \operatorname{Ar}^{+}\left(3 p^{-1}\right)-\mathrm{Ar} .
$$

Then, the delayed probe pulse interrupts the dissociation at $t_{\mathrm{d}}$ by ionizing to a doubly charged state ( $c f$. Fig. 16),

$$
\text { Probe: } \operatorname{Ar}^{+}\left(3 p^{-1}\right)-\operatorname{Ar} \stackrel{\mathrm{XUV}}{\longrightarrow} \operatorname{Ar}^{+}\left(3 p^{-1}\right)-\operatorname{Ar}^{+}\left(3 p^{-1}\right) \text {. }
$$

The overall KER is given by the kinetic energy accumulated on the initial $\operatorname{Ar}^{+}\left(3 p^{-1}\right)-\operatorname{Ar}$ and the final $\operatorname{Ar}^{+}\left(3 p^{-1}\right)-$ $\operatorname{Ar}^{+}\left(3 p^{-1}\right) \mathrm{PEC}$,

$$
\begin{aligned}
\mathrm{KER}= & E_{\text {init }}\left(R_{\mathrm{eq}}\right)-E_{\text {init }}\left[R\left(t_{\mathrm{d}}\right)\right]+E_{\text {final }}\left[R\left(t_{\mathrm{d}}\right)\right] \\
& -E_{\text {final }}(R \rightarrow \infty) .
\end{aligned}
$$

For large delays $\left|t_{\mathrm{d}}\right| \rightarrow \infty, E_{\text {final }}\left[R\left(\left|t_{\mathrm{d}}\right| \rightarrow \infty\right)\right]=E_{\text {final }}(R \rightarrow \infty)$ and no KER is accumulated on the final $\operatorname{Ar}^{+}\left(3 p^{-1}\right)-\operatorname{Ar}^{+}\left(3 p^{-1}\right)$ curve. Thus, the asymptotic KER of $\sim 1.0 \mathrm{eV}$ of $\mathrm{Ar}^{+}+\mathrm{Ar}^{+}$ions

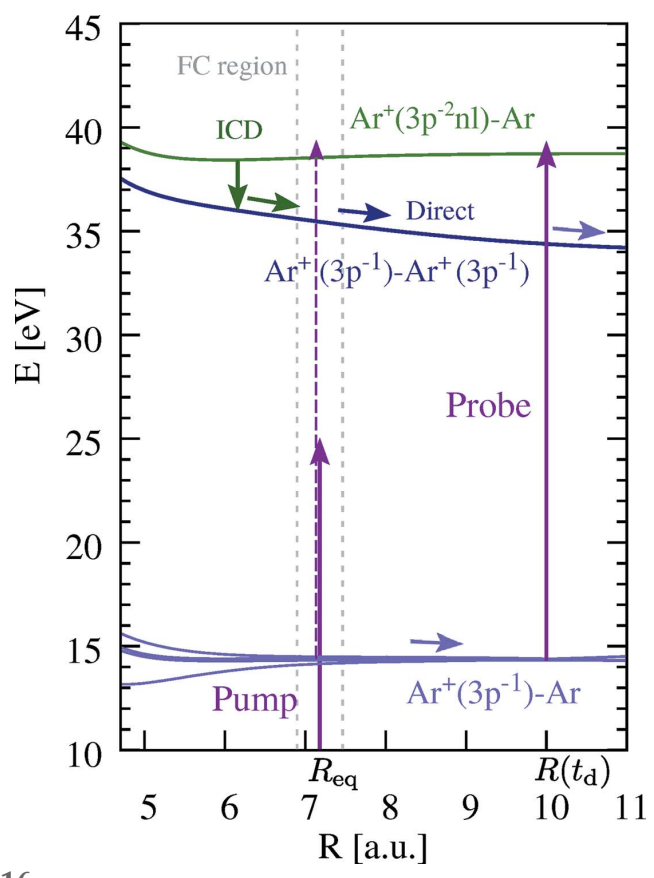

Figure 16

PECs of the argon dimer between 10 and $45 \mathrm{eV}$. The potential energy $E$ is given with respect to the $\mathrm{Ar}-\mathrm{Ar}$ ground state. For simplification, only a single $\mathrm{PEC}$ is plotted to represent $\operatorname{Ar}^{+}\left(3 p^{-2} n l\right)-\operatorname{Ar}$ (dark-green) and $\operatorname{Ar}^{+}\left(3 p^{-1}\right)-\operatorname{Ar}^{+}\left(3 p^{-1}\right)$ (blue) states. All curves are taken from Stoychev et al. (2008) and Miteva et al. (2014). [Reprinted with the permission of AIP Publishing.] The purple vertical arrows indicate photons of $\hbar \omega=$ $24.8 \mathrm{eV}$. The dashed arrow visualizes the absorption of a second photon in the pump pulse. The Franck-Condon (FC) region lies within the two vertical dashed gray lines. The figure is taken from Schmid (2018).

is a signature of the $\operatorname{Ar}^{+}\left(3 p^{-1}\right)-\operatorname{Ar}$ precursor state. The asymptotic limit is reached at large delays $\left|t_{\mathrm{d}}\right| \geq 2 \mathrm{ps}$, which corresponds to a slow dissociation of the dimer. The visibility of the signal over at least \pm 2 ps confirms the pump-probe capability over a large delay range and implies that the setup allows long-lasting dynamics to be traced.

The results presented in Sections 5.4 and 5.5 demonstrate that the in-line XUV split-delay and focusing optics opens up new opportunities for time-resolved electron-ion coincidence spectroscopy at FLASH2.

\section{Future upgrades}

An IR pump-probe laser is the next upgrade to the FLASH2 facility (FLASH2, 2018). It is also connected to the REMI endstation. The $800 \mathrm{~nm}$ laser system is OPCPA based and in the final stage will deliver pulses of sub-20 fs duration in burstmode $(10 \mathrm{~Hz}$ bursts with an intra-burst repetition rate of $50 \mathrm{kHz}$ initially). The individual pulses are foreseen to have energies of up to $0.5 \mathrm{~mJ}$. The timing jitter between FEL and IR pulses is initially $<50 \mathrm{fs}$ (RMS). With tight focusing, peak IR intensities of $>10^{15} \mathrm{~W} \mathrm{~cm}^{-2}$ should be attainable. First experiments at the REMI endstation using the IR pumpprobe laser were performed in the end of 2018. The incoupling mirror for the IR laser is installed in the last section of the REMI beamline (colored light-red in Fig. 9). A two-inch 
silver-coated plane mirror is mounted on a precision rotational feed-through, which is installed on a triaxial $x y z$-manipulator. To overlap the FEL and the IR beam co-linearly, the IR beam is deflected by $90^{\circ}$. The IR incoupling mirror has a hole of $4 \mathrm{~mm}$ diameter for the focused FEL beam to pass through. The IR laser is focused outside of the vacuum by a lens with a focal length of $40 \mathrm{~cm}$. The differential pumping tube between IR incoupling and REMI interaction chamber (4th tube in Fig. 9) has an inner diameter of $22 \mathrm{~mm}$. Thus, an IR beam of $\leq 35 \mathrm{~mm}$ diameter at the position of the lens can pass through the tube without clipping.

In the near future, in cooperation with DESY and the Leibniz University of Hanover, a high harmonic generation (HHG) source will also be integrated into the REMI beamline. This will further extend the portfolio of available radiation sources and allow for two-color VUV-XUV pump-probe spectroscopy.

Furthermore, the in-line geometry of the XUV focusing optics leaves room to re-use the FEL beam in a downstream experiment taking out the photon dump ( $c f$. Section 4.3). For example, a grating spectrometer operating on a shot-to-shot level could be useful to correlate the REMI data to the spectra of individual FEL pulses.

The next upgrade of the REMI apparatus itself will include a modular jet system. It will be mounted on rails and thus enables to easily switch between different sample injection systems.

\section{Summary}

In this contribution, the reaction microscope (REMI) endstation at beamline FL26 of the free-electron laser FLASH2 has been presented. The instrument is dedicated to time-resolved AMO-physics experiments on small quantum systems like atoms, molecules and small clusters by exploiting multi-particle coincidence spectroscopy. The sample injection, the design of the spectrometer, the functionality of the timeand position-sensitive detectors, the vacuum requirements and the data acquisition of the REMI were introduced. The design and the key parts of an in-line XUV split-delay and focusing optics were presented. The optics is based on grazing-incidence mirrors. With this the fast wavelength tunability of the FLASH2 variable-gap undulators can be efficiently exploited in XUV-XUV pump-probe experiments. A dedicated beamline section allows the FEL beam to be monitored and to be aligned before it enters the mirror chamber and the REMI. The beamline also serves as a differential pumping section to maintain UHV conditions of $\leq 10^{-11}$ mbar in the REMI interaction chamber. In the design of the beamline, special emphasis has been put on stray-light suppression, which is essential to perform multi-particle coincidence experiments at an FEL. Commissioning results demonstrate in particular the working capability of the in-line XUV split-delay and focusing optics. The endstation will be connected to the FLASH2 pump-probe laser system, which will enable XUV-IR pumpprobe experiments in the near future. Further upgrades will contain an HHG source and a modular jet system.

\section{Acknowledgements}

The authors thank the entire FLASH team including the accelerator, photon diagnostics and beamline groups. We are particularly grateful to C. Kaiser from the MPIK Heidelberg who helped in setting up the endstation. We especially acknowledge M. Brachmanski from FLASH for his support during the setup phase. Thanks also to B. Keitel, E. PlönjesPalm, T. Wodzinski and M. Ruiz Lopez who helped with operating the wavefront sensor.

\section{Funding information}

Funding for this research was provided by: Volkswagen Foundation (a Peter Paul Ewald Fellowship scholarship to KS).

\section{References}

Ackermann, W., Asova, G., Ayvazyan, V., Azima, A., Baboi, N., Bähr, J., Balandin, V., Beutner, B., Brandt, A., Bolzmann, A., Brinkmann, R., Brovko, O. I., Castellano, M., Castro, P., Catani, L., Chiadroni, E., Choroba, S., Cianchi, A., Costello, J. T., Cubaynes, D., Dardis, J., Decking, W., Delsim-Hashemi, H., Delserieys, A., Di Pirro, G., Dohlus, M., Düsterer, S., Eckhardt, A., Edwards, H. T., Faatz, B., Feldhaus, J., Flöttmann, K., Frisch, J., Fröhlich, L., Garvey, T., Gensch, U., Gerth, Ch., Görler, M., Golubeva, N., Grabosch, H., Grecki, M., Grimm, O., Hacker, K., Hahn, U., Han, J. H., Honkavaara, K., Hott, T., Hüning, M., Ivanisenko, Y., Jaeschke, E., Jalmuzna, W., Jezynski, T., Kammering, R., Katalev, V., Kavanagh, K., Kennedy, E. T., Khodyachykh, S., Klose, K., Kocharyan, V., Körfer, M., Kollewe, M., Koprek, W., Korepanov, S., Kostin, D., Krassilnikov, M., Kube, G., Kuhlmann, M., Lewis, C. L. S., Lilje, L., Limberg, T., Lipka, D., Löhl, F., Luna, H., Luong, M., Martins, M., Meyer, M., Michelato, P., Miltchev, V., Möller, W. D., Monaco, L., Müller, W. F. O., Napieralski, O., Napoly, O., Nicolosi, P., Nölle, D., Nuñez, T., Oppelt, A., Pagani, C., Paparella, R., Pchalek, N., Pedregosa-Gutierrez, J., Petersen, B., Petrosyan, B., Petrosyan, G., Petrosyan, L., Pflüger, J., Plönjes, E., Poletto, L., Pozniak, K., Prat, E., Proch, D., Pucyk, P., Radcliffe, P., Redlin, H., Rehlich, K., Richter, M., Roehrs, M., Roensch, J., Romaniuk, R., Ross, M., Rossbach, J., Rybnikov, V., Sachwitz, M., Saldin, E. L., Sandner, W., Schlarb, H., Schmidt, B., Schmitz, M., Schmüser, P., Schneider, J. R., Schneidmiller, E. A., Schnepp, S., Schreiber, S., Seidel, M., Sertore, D., Shabunov, A. V., Simon, C., Simrock, S., Sombrowski, E., Sorokin, A. A., Spanknebel, P., Spesyvtsev, R., Staykov, L., Steffen, B., Stephan, F., Stulle, F., Thom, H., Tiedtke, K., Tischer, M., Toleikis, S., Treusch, R., Trines, D., Tsakov, I., Vogel, E., Weiland, T., Weise, H., Wellhöfer, M., Wendt, M., Will, I., Winter, A., Wittenburg, K., Wurth, W., Yeates, P., Yurkov, M. V., Zagorodnov, I. \& Zapfe, K. (2007). Nat. Photon. 1, 336-342.

Benvenuti, C., Chiggiato, P., Pinto, P. C., Santana, A. E., Hedley, T., Mongelluzzo, A., Ruzinov, V. \& Wevers, I. (1999). Vacuum, 53, 219 225.

Bittner, L., Maschinenphysik, D., Feldhaus, J., Hahn, U., Hesse, M., Jastrow, U., Kocharyan, V., Radcliffe, P., Saldin, E., Schneidmiller, E., Tiedtke, K., Timmann, B., Treusch, R., Bargen, N., Yurkov, M., Brovko, O., Kharlamov, D., Lokhmatov, V., Matyushevskiy, E. \& Syresin, E. (2007). Proceedings of the 29th International FreeElectron Laser Conference (FEL2007), 26-31 August 2007, Novosibirsk, Russia.

Braune, M., Brenner, G., Dziarzhytski, S., Juranić, P., Sorokin, A. \& Tiedtke, K. (2016). J. Synchrotron Rad. 23, 10-20.

Callegari, C., Prince, K. C. \& Ueda, K. (2016). Synchrotron Radiat. News, 29(3), 21-25.

CERN (2018). ROOT, https://root.cern.ch/. Last request: 22/12/2018. 
Dörner, R., Mergel, V., Jagutzki, O., Spielberger, L., Ullrich, J., Moshammer, R. \& Schmidt-Böcking, H. (2000). Phys. Rep. 330, 95192.

Erk, B., Müller, J. P., Bomme, C., Boll, R., Brenner, G., Chapman, H. N., Correa, J., Düsterer, S., Dziarzhytski, S., Eisebitt, S., Graafsma, H., Grunewald, S., Gumprecht, L., Hartmann, R., Hauser, G., Keitel, B., von Korff Schmising, C., Kuhlmann, M., Manschwetus, B., Mercadier, L., Müller, E., Passow, C., Plönjes, E., Ramm, D., Rompotis, D., Rudenko, A., Rupp, D., Sauppe, M., Siewert, F., Schlosser, D., Strüder, L., Swiderski, A., Techert, S., Tiedtke, K., Tilp, T., Treusch, R., Schlichting, I., Ullrich, J., Moshammer, R., Möller, T. \& Rolles, D. (2018). J. Synchrotron Rad. 25, 1529-1540.

Faatz, B., Plönjes, E., Ackermann, S., Agababyan, A., Asgekar, V., Ayvazyan, V., Baark, S., Baboi, N., Balandin, V., Bargen, N., Bican, Y., Bilani, O., Bödewadt, J., Böhnert, M., Böspflug, R., Bonfigt, S., Bolz, H., Borges, F., Borkenhagen, O., Brachmanski, M., Braune, M., Brinkmann, A., Brovko, O., Bruns, T., Castro, P., Chen, J., Czwalinna, M. K., Damker, H., Decking, W., Degenhardt, M., Delfs, A., Delfs, T., Deng, H., Dressel, M., Duhme, H., Düsterer, S., Eckoldt, H., Eislage, A., Felber, M., Feldhaus, J., Gessler, P., Gibau, M., Golubeva, N., Golz, T., Gonschior, J., Grebentsov, A., Grecki, M., Grün, C., Grunewald, S., Hacker, K., Hänisch, L., Hage, A., Hans, T., Hass, E., Hauberg, A., Hensler, O., Hesse, M., Heuck, K., Hidvegi, A., Holz, M., Honkavaara, K., Höppner, H., Ignatenko, A., Jäger, J., Jastrow, U., Kammering, R., Karstensen, S., Kaukher, A., Kay, H., Keil, B., Klose, K., Kocharyan, V., Köpke, M., Körfer, M., Kook, W., Krause, B., Krebs, O., Kreis, S., Krivan, F., Kuhlmann, J., Kuhlmann, M., Kube, G., Laarmann, T., Lechner, C., Lederer, S., Leuschner, A., Liebertz, D., Liebing, J., Liedtke, A., Lilje, L., Limberg, T., Lipka, D., Liu, B., Lorbeer, B., Ludwig, K., Mahn, H., Marinkovic, G., Martens, C., Marutzky, F., Maslocv, M., Meissner, D., Mildner, N., Miltchev, V., Molnar, S., Mross, D., Müller, F., Neumann, R., Neumann, P., Nölle, D., Obier, F., Pelzer, M., Peters, H., Petersen, K., Petrosyan, A., Petrosyan, G., Petrosyan, L., Petrosyan, V., Petrov, A., Pfeiffer, S., Piotrowski, A., Pisarov, Z., Plath, T., Pototzki, P., Prandolini, M. J., Prenting, J., Priebe, G., Racky, B., Ramm, T., Rehlich, K., Riedel, R., Roggli, M., Röhling, M., Rönsch-Schulenburg, J., Rossbach, J., Rybnikov, V., Schäfer, J., Schaffran, J., Schlarb, H., Schlesselmann, G., Schlösser, M., Schmid, P., Schmidt, C., Schmidt-Föhre, F., Schmitz, M., Schneidmiller, E., Schöps, A., Scholz, M., Schreiber, S., Schütt, K., Schütz, U., SchulteSchrepping, H., Schulz, M., Shabunov, A., Smirnov, P., Sombrowski, E., Sorokin, A., Sparr, B., Spengler, J., Staack, M., Stadler, M., Stechmann, C., Steffen, B., Stojanovic, N., Sychev, V., Syresin, E., Tanikawa, T., Tavella, F., Tesch, N., Tiedtke, K., Tischer, M., Treusch, R., Tripathi, S., Vagin, P., Vetrov, P., Vilcins, S., Vogt, M., Wagner, A. Z., Wamsat, T., Weddig, H., Weichert, G., Weigelt, H., Wentowski, N., Wiebers, C., Wilksen, T., Willner, A., Wittenburg, K., Wohlenberg, T., Wortmann, J., Wurth, W., Yurkov, M., Zagorodnov, I. \& Zemella, J. (2016). New J. Phys. 18, 062002.

Fechner, L. (2014). High-Resolution Experiments on Strong-Field Ionization of Atoms and Molecules. Springer International Publishing.

Feldhaus, J., Krikunova, M., Meyer, M., Möller, T., Moshammer, R., Rudenko, A., Tschentscher, T. \& Ullrich, J. (2013). J. Phys. B At. Mol. Opt. Phys. 46, 164002.

FLASH2 (2018). FLASH2 pump-probe laser system, http://photonscience.desy.de/facilities/flash/beamlines/optical_laser_systems/ index_eng.html. Last request: 12/04/2018.

Fritzsche, S., Grum-Grzhimailo, A. N., Gryzlova, E. V. \& Kabachnik, N. M. (2008). J. Phys. B At. Mol. Opt. Phys. 41, 165601.

Gallagher, J. W., Brion, C. E., Samson, J. A. R. \& Langhoff, P. W. (1988). J. Phys. Chem. Ref. Data, 17, 9-153.

Gerken, N., Klumpp, S., Sorokin, A. A., Tiedtke, K., Richter, M., Bürk, V., Mertens, K., Juranić, P. \& Martins, M. (2014). Phys. Rev. Lett. 112, 213002.
Grum-Grzhimailo, A. N., Gryzlova, E. V., Strakhova, S. I., Kabachnik, N. M. \& Fritzsche, S. (2009). J. Phys. Conf. Ser. 194, 012004.

GSI (2018). Go4, http://go4.gsi.de/. Last request: 22/12/2018.

Henke, B. L., Gullikson, E. M. \& Davis, J. C. (1993). At. Data Nucl. Data Tables, 54, 181-342.

Hikosaka, Y., Fushitani, M., Matsuda, A., Tseng, C.-M., Hishikawa, A., Shigemasa, E., Nagasono, M., Tono, K., Togashi, T., Ohashi, H., Kimura, H., Senba, Y., Yabashi, M. \& Ishikawa, T. (2010). Phys. Rev. Lett. 105, 133001.

Hunstig, M. (2017). Actuators, 6, 7.

Jagutzki, O., Cerezo, A., Czasch, A., Dörner, R., Hattas, M., Huang, M., Mergel, V., Spillmann, U., Ullmann-Pfleger, K., Weber, T., Schmidt-Böcking, H. \& Smith, G. (2002a). IEEE Trans. Nucl. Sci. 49, 2477-2483.

Jagutzki, O., Mergel, V., Ullmann-Pfleger, K., Spielberger, L., Spillmann, U., Dörner, R. \& Schmidt-Böcking, H. (2002b). Nucl. Instrum. Methods Phys. Res. A, 477, 244-249.

Jiang, Y. H., Rudenko, A., Herrwerth, O., Foucar, L., Kurka, M., Kühnel, K. U., Lezius, M., Kling, M. F., van Tilborg, J., Belkacem, A., Ueda, K., Düsterer, S., Treusch, R., Schröter, C. D., Moshammer, R. \& Ullrich, J. (2010a). Phys. Rev. Lett. 105, 263002.

Jiang, Y. H., Rudenko, A., Pérez-Torres, J., Herrwerth, O., Foucar, L., Kurka, M., Kühnel, K. U., Toppin, M., Plésiat, E., Morales, F., Martín, F., Lezius, M., Kling, M. F., Jahnke, T., Dörner, R., SanzVicario, J. L., van Tilborg, J., Belkacem, A., Schulz, M., Ueda, K., Zouros, T., Düsterer, S., Treusch, R., Schröter, C. D., Moshammer, R. \& Ullrich, J. (2010b). Phys. Rev. A, 81, 051402.

Jousten, K. (2010). Wutz Handbuch Vakuumtechnik. Praxis, 10th ed. Wiesbaden: Vieweg + Teubner.

Keitel, B., Plönjes, E., Kreis, S., Kuhlmann, M., Tiedtke, K., Mey, T., Schäfer, B. \& Mann, K. (2016). J. Synchrotron Rad. 23, 43-49.

Kheifets, A. S. (2007). J. Phys. B At. Mol. Opt. Phys. 40, F313F318.

Krikunova, M., Adolph, M., Gorkhover, T., Rupp, D., Schorb, S., Bostedt, C., Roling, S., Siemer, B., Mitzner, R., Zacharias, H. \& Möller, T. (2012). J. Phys. B At. Mol. Opt. Phys. 45, 105101.

Kurka, M., Feist, J., Horner, D. A., Rudenko, A., Jiang, Y. H., Kühnel, K. U., Foucar, L., Rescigno, T. N., McCurdy, C. W., Pazourek, R., Nagele, S., Schulz, M., Herrwerth, O., Lezius, M., Kling, M. F., Schöffler, M., Belkacem, A., Düsterer, S., Treusch, R., Schneider, B. I., Collins, L. A., Burgdörfer, J., Schröter, C. D., Moshammer, R. \& Ullrich, J. (2010). New J. Phys. 12, 073035.

Kurka, M., Rudenko, A., Foucar, L., Kühnel, K. U., Jiang, Y. H., Ergler, T., Havermeier, T., Smolarski, M., Schössler, S., Cole, K., Schöffler, M., Dörner, R., Gensch, M., Düsterer, S., Treusch, R., Fritzsche, S., Grum-Grzhimailo, A. N., Gryzlova, E. V., Kabachnik, N. M., Schröter, C. D., Moshammer, R. \& Ullrich, J. (2009). J. Phys. B At. Mol. Opt. Phys. 42, 141002.

Lampton, M., Siegmund, O. \& Raffanti, R. (1987). Rev. Sci. Instrum. 58, 2298-2305.

Magrakvelidze, M., Herrwerth, O., Jiang, Y. H., Rudenko, A., Kurka, M., Foucar, L., Kühnel, K. U., Kübel, M., Johnson, N. G., Schröter, C. D., Düsterer, S., Treusch, R., Lezius, M., Ben-Itzhak, I., Moshammer, R., Ullrich, J., Kling, M. F. \& Thumm, U. (2012). Phys. Rev. A, 86, 013415.

Meister, S. (2016). Master thesis, University of Heidelberg, Germany. Miteva, T., Chiang, Y.-C., Kolorenč, P., Kuleff, A., Gokhberg, K. \& Cederbaum, L. S. (2014). J. Chem. Phys. 141, 064307.

Moshammer, R., Jiang, Y. H., Foucar, L., Rudenko, A., Ergler, T., Schröter, C. D., Lüdemann, S., Zrost, K., Fischer, D., Titze, J., Jahnke, T., Schöffler, M., Weber, T., Dörner, R., Zouros, T., Dorn, A., Ferger, T., Kühnel, K. U., Düsterer, S., Treusch, R., Radcliffe, P., Plönjes, E. \& Ullrich, J. (2007). Phys. Rev. Lett. 98, 203001.

Moshammer, R. \& Schnorr, K. (2014). Molecular Physics and GasPhase Chemistry with Free-Electron Lasers, pp. 1-29. Cham: Springer International Publishing. 
Moshammer, R., Unverzagt, M., Schmitt, W., Ullrich, J. \& SchmidtBöcking, H. (1996). Nucl. Instrum. Methods Phys. Res. B, 108, 425445.

Noll, T., Holldack, K., Reichardt, G., Schwarzkopf, O. \& Zeschke, T. (2009). Precis. Eng. 33, 291-304.

Plönjes, E., Faatz, B., Kuhlmann, M. \& Treusch, R. (2016). AIP Conf. Proc. 1741, 020008.

Pontius, N., Beye, M., Trabant, C., Mitzner, R., Sorgenfrei, F., Kachel, T., Wöstmann, M., Roling, S., Zacharias, H., Ivanov, R., Treusch, R., Buchholz, M., Metcalf, P., Schüssler-Langeheine, C. \& Föhlisch, A. (2018). Struct. Dyn. 5, 054501.

Richter, M., Amusia, M. Y., Bobashev, S. V., Feigl, T., Juranić, P. N., Martins, M., Sorokin, A. A. \& Tiedtke, K. (2009). Phys. Rev. Lett. 102, 163002.

Rudenko, A., Jiang, Y. H., Kurka, M., Kühnel, K. U., Foucar, L., Herrwerth, O., Lezius, M., Kling, M. F., Schröter, C. D., Moshammer, R. \& Ullrich, J. (2010). J. Phys. B At. Mol. Opt. Phys. 43, 194004.

Sauppe, M., Rompotis, D., Erk, B., Bari, S., Bischoff, T., Boll, R., Bomme, C., Bostedt, C., Dörner, S., Düsterer, S., Feigl, T., Flückiger, L., Gorkhover, T., Kolatzki, K., Langbehn, B., Monserud, N., Müller, E., Müller, J. P., Passow, C., Ramm, D., Rolles, D., Schubert, K., Schwob, L., Senfftleben, B., Treusch, R., Ulmer, A., Weigelt, H., Zimbalski, J., Zimmermann, J., Möller, T. \& Rupp, D. (2018). J. Synchrotron Rad. 25, 1517-1528.

Schmid, G. (2018). PhD thesis, University of Heidelberg, Germany. Schnorr, K. (2014). PhD thesis, University of Heidelberg, Germany.

Schnorr, K., Senftleben, A., Kurka, M., Rudenko, A., Foucar, L., Schmid, G., Broska, A., Pfeifer, T., Meyer, K., Anielski, D., Boll, R., Rolles, D., Kübel, M., Kling, M. F., Jiang, Y. H., Mondal, S., Tachibana, T., Ueda, K., Marchenko, T., Simon, M., Brenner, G., Treusch, R., Scheit, S., Averbukh, V., Ullrich, J., Schröter, C. D. \& Moshammer, R. (2013). Phys. Rev. Lett. 111, 093402.

Schnorr, K., Senftleben, A., Kurka, M., Rudenko, A., Schmid, G., Pfeifer, T., Meyer, K., Kübel, M., Kling, M. F., Jiang, Y. H., Treusch,
R., Düsterer, S., Siemer, B., Wöstmann, M., Zacharias, H., Mitzner, R., Zouros, T. J. M., Ullrich, J., Schröter, C. D. \& Moshammer, R. (2014). Phys. Rev. Lett. 113, 073001.

Scoles, G. (1988). Atomic and Molecular Beam Methods: 1. Atomic and Molecular Beam Methods. Oxford University Press.

Sorokin, A. A., Bobashev, S. V., Feigl, T., Tiedtke, K., Wabnitz, H. \& Richter, M. (2007a). Phys. Rev. Lett. 99, 213002.

Sorokin, A. A., Wellhöfer, M., Bobashev, S. V., Tiedtke, K. \& Richter, M. (2007b). Phys. Rev. A, 75, 051402.

Stoychev, S. D., Kuleff, A. I., Tarantelli, F. \& Cederbaum, L. S. (2008). J. Chem. Phys. 128, 014307.

Tiedtke, K., Azima, A., von Bargen, N., Bittner, L., Bonfigt, S., Düsterer, S., Faatz, B., Frühling, U., Gensch, M., Gerth, C., Guerassimova, N., Hahn, U., Hans, T., Hesse, M., Honkavaar, K., Jastrow, U., Juranic, P., Kapitzki, S., Keitel, B., Kracht, T., Kuhlmann, M., Li, W. B., Martins, M., Núñez, T., Plönjes, E., Redlin, H., Saldin, E. L., Schneidmiller, E. A., Schneider, J. R., Schreiber, S., Stojanovic, N., Tavella, F., Toleikis, S., Treusch, R., Weigelt, H., Wellhöfer, M., Wabnitz, H., Yurkov, M. V. \& Feldhaus, J. (2009). New J. Phys. 11, 023029.

Ullrich, J., Moshammer, R., Dorn, A., Dörner, R., Schmidt, L. \& Schmidt-Böcking, H. (2003). Rep. Prog. Phys. 66, 1463-1545.

Usenko, S., Przystawik, A., Jakob, M. A., Lazzarino, L. L., Brenner, G., Toleikis, S., Haunhorst, C., Kip, D. \& Laarmann, T. (2017). Nat. Commun. 8, 15626.

Wiley, W. C. \& McLaren, I. H. (1955). Rev. Sci. Instrum. 26, 11501157.

Wiza, J. (1979). Nucl. Instrum. Methods, 162, 587-601.

Yabashi, M., Tanaka, H., Tanaka, T., Tomizawa, H., Togashi, T., Nagasono, M., Ishikawa, T., Harries, J. R., Hikosaka, Y., Hishikawa, A., Nagaya, K., Saito, N., Shigemasa, E., Yamanouchi, K. \& Ueda, K. (2013). J. Phys. B At. Mol. Opt. Phys. 46, 164001.

Yeh, J. J. \& Lindau, I. (1985). At. Data Nucl. Data Tables, 32, 1-155. Zewail, A. H. (1988). Science, 242, 1645-1653.

Zewail, A. H. (2000). J. Phys. Chem. A, 104, 5660-5694. 